\title{
A Multiband Millimeter-Wave Two- Dimensional Array Based on Enhanced Franklin Antenna for 5G Wireless Systems
}

\author{
Syeda Fizzah Jilani, Student Member, IEEE, and Akram Alomainy, Senior Member, IEEE
}

\begin{abstract}
This letter presents a novel and compact antenna array suggesting geometrical modifications in the concept of collinear arrays at millimeter-wave (MMW) spectrum. The extension of the standard narrowband Franklin linear antenna array in a compact two-dimensional (2D) assembly is proposed and multiband response is accomplished in this work which fulfills demands of future $5 \mathrm{G}$ systems. The proposed $2 \mathrm{D}$ antenna array is designed to operate at licensed frequency bands of 28GHz and 37-39 GHz recommended by Federal Communications Commission (FCC) for 5G wireless networks, as well as at 33$\mathrm{GHz}$ for satellite communications and radio navigation. Measurements validate the triple-band response at the desired frequencies of operation. The proposed antenna array also offers distinguishing performance in terms of high gain and significant efficiency above $70 \%$ in all the MMW operating bands.
\end{abstract}

Index Terms-5G, collinear, directivity, Franklin, multiband.

\section{INTRODUCTION}

$\mathrm{F}$ IFTH Generation (5G) of mobile networks is anticipated to bring an effective solution for bandwidth scarcity projected by the exponential growth of wireless devices and ever-increasing demand of mobile applications $[1,2]$. As the current wireless spectrum is drastically losing its free space, it is highly important to introduce alternative choices of frequency bands that can support the ongoing progress of wireless networks. The unused MMW spectrum has drawn huge attention in realizing the aforementioned $5 \mathrm{G}$ vision, and is capable of facilitating advanced wireless applications which cannot be incorporated in a limited bandwidth of current wireless bands. The intended MMW infrastructure for $5 \mathrm{G}$ mobile channels will be able to support much better data speeds, higher aggregate capacity, minimal latency, lower infrastructure cost, and many new capabilities due to increased bandwidth [3]. Another advantage of shorter wavelengths of MMWs from installation perspectives is the reduced antenna size which allows incorporation of multiple patches or arrays

Special thanks to National University of Sciences and Technology for providing financial support to this research. Authors are also grateful to Queen Mary University of London for the research facilities.

S. F. Jilani and A. Alomainy are with the School of Electronic Engineering and Computer Science, Queen Mary University of London, London E1 4NS, United Kingdom, and also with the National University of Sciences and Technology, Pakistan (e-mail: s.f.jilani@qmul.ac.uk and a.alomainy@qmul.ac.uk). in a compact handset or indoor basestation (BS) to effectively increase the gain performance [4]. Although MMWs can be easily employed for short-range indoor setups, a number of challenges are involved in its use for outdoor systems. Many limitations needed to be resolved to realize an entirely new architecture based on MMWs, such as propagation losses, atmospheric absorptions, rain fades, shadowing, and high attenuation levels due to obstacles [5]. To overcome these challenges, intensive efforts have been carried out to establish innovative wireless models suggesting the implementation of high gain antennas in dense picocells and femtocells to facilitate low propagation losses and frequency reuse.

FCC has taken an initiative to approve licensed bands of 28$\mathrm{GHz}, 37-\mathrm{GHz}, 39-\mathrm{GHz}$, and an unlicensed spectrum of 64-71 $\mathrm{GHz}$, as the prospective candidates for $5 \mathrm{G}$ networks [6]. This progress has directed the attention of antenna designers to suggest efficient and high gain antenna systems, capable of simultaneous operation in the proposed $5 \mathrm{G}$ bands to enhance the spectrum availability for the cellular network providers. High gain and directivity requirements could be accomplished by integrating multiple antenna elements into arrays or grid assemblies, where individual radiating beams of each antenna are combined to provide a collective beam with high directivity. Several efforts have been made to fulfill $5 \mathrm{G}$ requirements in novel antenna geometries as well as array configurations at MMWs, especially at 28- and 38-GHz [711]. In most of the reported antennas, the desired performance is associated with design complexities, usually due to multilayer structures which leads to high fabrication cost. In this research work, an attempt to design a multiband antenna array has been made at 5G MMW bands of 28-GHz and 37-39 $\mathrm{GHz}$ as well as $33-\mathrm{GHz}$ for satellite communications along with advantages of structural simplicity, planar geometry, ease of fabrication and relatively lower complexity.

The conventional Franklin antenna is based on collinear array (CoA) principle, composed of a series of radiating elements provided with an in-phase feeding and offers highgain performance [12-14]. In a Franklin array, two thin nonradiating transmission lines between two adjacent halfwavelength $(\lambda / 2)$ long patches are folded to establish $180^{\circ}$ phase shift, and create a symmetrical quarter-wave $(\lambda / 4)$ phasing stub arrangement with the minimum parasitic discontinuity at the junction of the patch and stub. The radiated beam is usually directed towards broadside direction 
over a narrow bandwidth [12-15]. A 2D assembly of CoA was introduced in [14] with advantages of compactness and high gain of $15.4 \mathrm{dBi}$ but restricted to a single-band operation. Several modifications of Franklin antenna arrays have also been reported to achieve high gain [16-18]; still the critical limitation is the narrowband and single frequency operation. This paper suggests a novel compact 2D array where the conventional CoA principle has been intentionally deviated in order to realize multiple resonances at MMW spectrum.

\section{Design Methodology and Fabrication}

A multiband MMW array based on enhanced Franklin array concept provided with 2D illustration is presented in this work for the first time. Antenna design is initiated by altering the parameters of array geometry with the objective of extending the concept of the Franklin array in order to generate multiple frequency bands, rather than a single resonant frequency. CoA is composed of a series of radiating patches and non-radiating stubs constituting a phase shift of $180^{\circ}$, as shown in fig.1 (a). The first geometrical modification made in this work is that the standard non-radiating stubs are modified into foldeddipole-like antennas, where the resonant frequency is controlled by the radiating length of the stubs. This transformation provides an ease to generate additional resonance in the same compact area with a compromise of slight detuning of main beam as the phasing network has been removed. Hence, each unit-cell consists of the rectangular patch, and the folded-dipole-like stub, as shown in fig. 1 (b), both radiating at different frequencies. Furthermore, the geometry of the standard linear Franklin array is extended in a 2D arrangement, in order to present a novel, highperformance, and compact antenna array. The $28-\mathrm{GHz}$ band is attained in the proposed geometry by using a stub length of $\lambda / 4$ (i.e., at $28-\mathrm{GHz}$ ) instead of $\lambda / 2$ as suggested in $[14,16]$, in fig. 1 (a). Then folded from the center point to construct a radiating element like a folded-dipole-antenna, in fig. 1 (b). In this way, an additional resonance is generated in the feeding network, which has been transformed into a folded-dipole-like antenna and is fed from the previous patch antenna, as shown by the current distribution in fig. 1 (c).

The patch antennas are designed based on $\lambda / 2$ of the resonant frequency mid-way between $33-\mathrm{GHz}$ and $38-\mathrm{GHz}$. It has been observed that a single resonance at $36.5-\mathrm{GHz}$ can provide a dual-band response by increasing the length of the patches positioned at the terminating edge of the array, by an amount denoted as $s$, shown in fig. 1 (d). The incremental length $s$ is introduced in the patch length $L_{p}$ of the terminating row, to appropriately adjust the wave propagation as the patches at the far edge of the grid are not interconnected. Parametric studies have shown that the $33-\mathrm{GHz}$ and $38-\mathrm{GHz}$ bands can be tuned by varying the parameter $L_{p}$ along the $y$ axis, i.e., the greater the length, the lower the resonant frequency will be. The distance between the centers of the two parallel patches is $\lambda$ (i.e., at $38-\mathrm{GHz}$ ) to lower the mutual coupling. Moreover, the standard unit-cell proposed in [16] consisting of a dipole arrangement of two patches of length $\lambda / 2$ and a $\lambda / 2$-long feeding circuitry in the center, has been fundamentally changed, which has resulted in an antenna array as a well-matched 2D assembly of patches and folded-dipolelike antennas. Each element (i.e., patch or folded-dipole) acts as a fully-matched standalone antenna to minimize parasitic discontinuities without any additional feeding network incorporated between antennas, and also each antenna is fed from the preceding antenna.

Microstrip line along with the stub of optimized dimensions is designed to feed the array from a single point. The simulation modelling of antenna array geometry along with the designed parameters, as well as the fabricated prototype are presented in figs. 1 (d) and (e), and the details of the design parameters are illustrated in Table 1. The CST Studio Suite software has been employed for the antenna modelling and numerical evaluations. Rogers RT/Duroid $5880\left(\varepsilon_{r}=2.2\right.$, and $\tan \delta=0.0009$ ) of height $h=0.8 \mathrm{~mm}$ has been used as a substrate material provided with metallic ground at the bottom. The $50-\Omega$ matched $K$-connector is also mounted on the feed line for the measurement process.

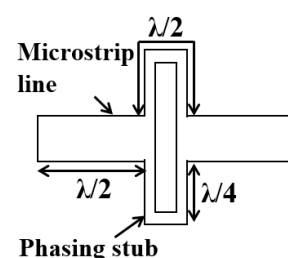

(a)

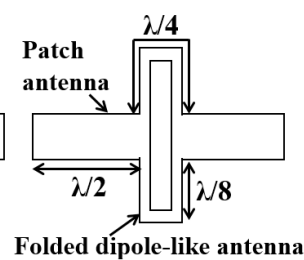

(b)

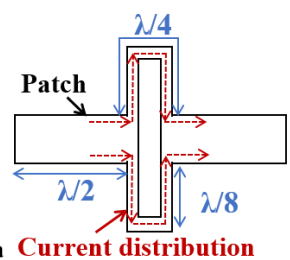

(c)

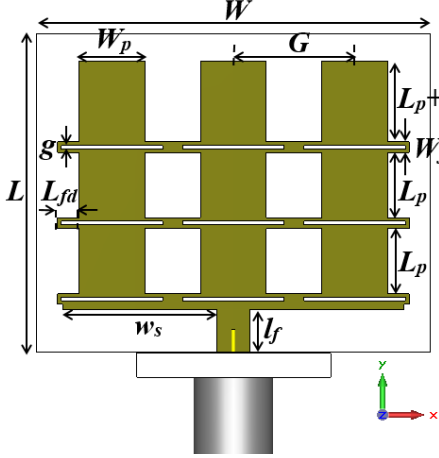

(d)

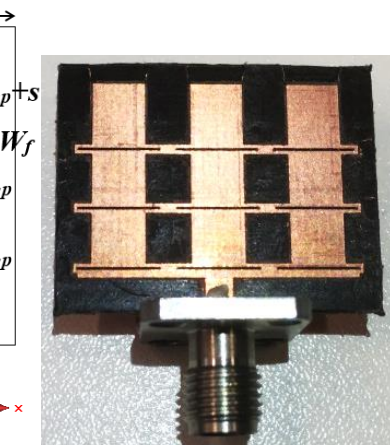

(e)
Fig. 1. The proposed MMW 2D antenna array: (a) Franklin array unit-cell; (b) array unit-cell; (c) array current distribution; (d) simulated prototype with the optimized dimensions; (e) fabricated antenna prototype.

TABLE I

Optimized Dimensions of the Proposed MMW ANTENNA ARRAY

$\begin{array}{cll}\text { Symbol } & \text { Parameters } & \mathbf{( m m )} \\ L_{p} & \text { Length of patch } & 4.3 \\ W_{p} & \text { Width of patch } & 4.34 \\ s & \text { Extended length of terminating patches } & 1.0 \\ G & \text { Gap between centres of two parallel patches } & 8.0 \\ L_{f d} & \text { Length of folded-dipole } & 1.4 \\ W_{f} & \text { Width of folded-dipole } & 0.7 \\ w_{s} & \text { Width of matching stub } & 10.15 \\ l_{f} & \text { Length of feed } & 2.8 \\ g & \text { Gap of folded-dipole } & 0.26 \\ L & \text { Length of array prototype } & 21.0 \\ W & \text { Width of array prototype } & 26.0\end{array}$

mm) 


\section{RESULTS AND Discussion}

The performance of the proposed antenna array is critically investigated by the parametric analysis, and further validated by measurements of the $s$-parameters, radiation pattern, and realized gain. Measurements of the antenna array show good agreement with the simulated results. However, some mismatches are also observed mainly due to the factors including fabrication intolerances, connector, and cable losses.

\section{A. Impedance Bandwidth}

Fig. 2 presents the measured reflection coefficient profile of the array validating the multiband response (i.e., 28-GHz, 33$\mathrm{GHz}$, and 38-GHz) referred in the simulation. Mutual coupling associated with the gap between the patches, dimensions of feed line, and gap of the folded-stub are important parameters to optimize the $S_{11}$ characteristics.

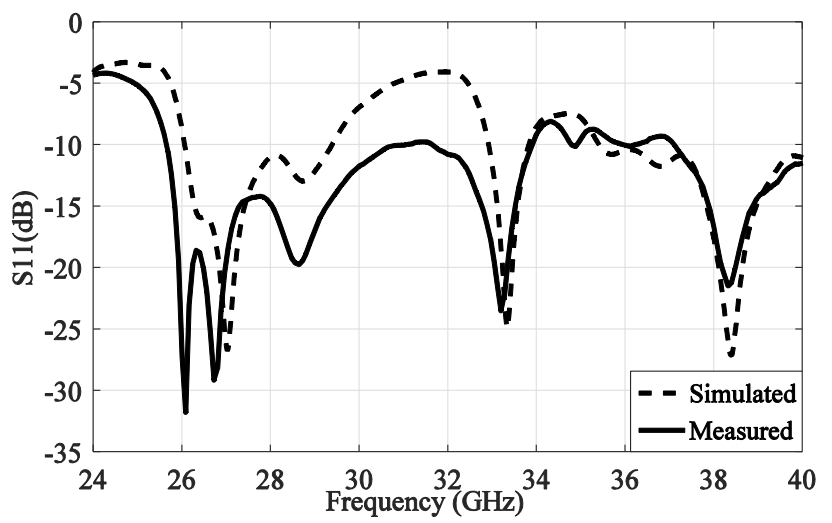

Fig. 2. Simulated and measured $S_{11}$ plot of the MMW 2D antenna array.

Parametric analysis is conducted to examine the parameters capable of tuning the resonant frequencies of the antenna array. Parametric study of fig. 3 (a) shows that $L_{p}$ is associated with frequency tuning of $33-\mathrm{GHz}$ and $38-\mathrm{GHz}$ bands, i.e., greater the length, lower the resonant frequency will be. It is important to modify $L_{p}$ of the patches at the terminating edge of the array to adjust the wave propagation as these patches are not interconnected by means of folded-dipole assembly. It has been observed that the increase in $L_{p}$ at terminating patches splits a single resonance at $36.5-\mathrm{GHz}$ (i.e., when $s=-$ $0.55 \mathrm{~mm}$ ) into a dual-band, where the $s$ is also capable of frequency tuning, as shown in fig. 3 (b).

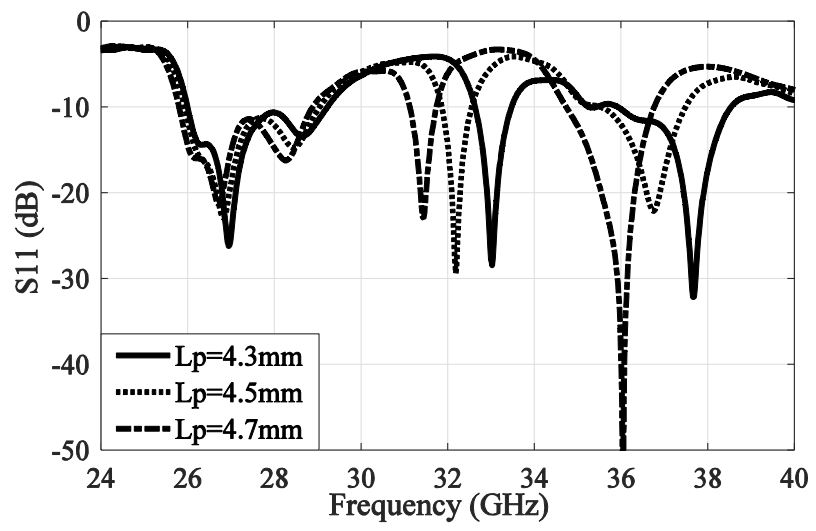

(a)

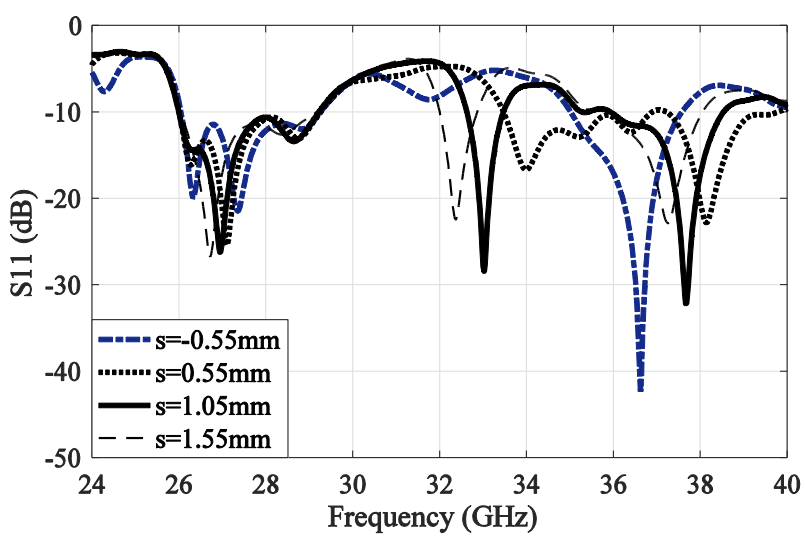

(b)

Fig. 3. Parametric analysis of the MMW 2D antenna array: (a) analysis by varying the length of patch $L_{p}$; (b) analysis with incremental length $s$.

\section{B. Radiation Pattern}

The absence of phasing network results in the main beam shift from $0^{\circ}$ of broadside direction in all the three bands of the proposed array. 5G communication will not be strictly line-of-sight (LoS) [19]. High directivity and gain requirements for $5 \mathrm{G}$ MMW communications are mainly to overcome path losses in both LOS and NLoS scenarios. The observed beam shift will not affect the performance of proposed antenna in NLoS links. Band 1 at $28-\mathrm{GHz}$ offers high directivity with maximum value at $29-\mathrm{GHz}$ where the main beam is at $50^{\circ}$. In $33-\mathrm{GHz}$ and $38-\mathrm{GHz}$ bands, the main beam is dispersed to some extent thus having lower directivity than $28-\mathrm{GHz}$ band. Fig. 4 presents the normalized radiation pattern of the proposed array at these three suggested bands.

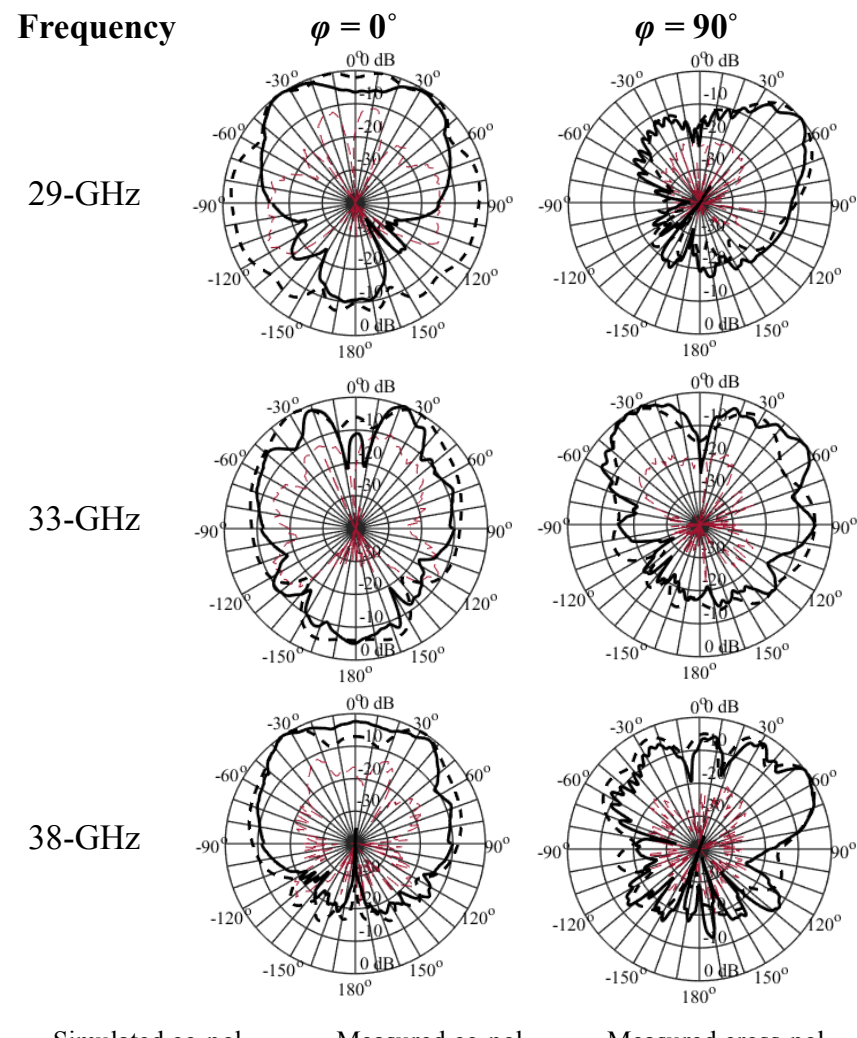

--- Simulated co-pol - Measured co-pol --- Measured cross-pol Fig. 4. Simulated and measured normalized radiation patterns (at $\varphi=0^{\circ}$ and $\varphi$ $=90^{\circ}$ ) of the MMW 2D antenna array at 29-, 33-, and 38-GHz bands. 


\section{Surface Current and Realized Gain}

Surface current distribution in fig. 6 (a) shows that at 29$\mathrm{GHz}$ a high current density is observed at the folded-dipole stubs. However, fig. 6 (b and c) shows current density is higher at patches then stubs, which explains 33-GHz and 38$\mathrm{GHz}$ bands are controlled by patch dimensions. Fig. 7 shows the simulated and measured realized gain profile of the proposed MMW antenna array. The measurements show that the peak gain of magnitude $13.5 \mathrm{dBi}$ is observed at $29-\mathrm{GHz}$, while the other bands also offer reasonable gain characteristics, i.e., $8.33 \mathrm{dBi}$ at $33-\mathrm{GHz}$, and $9.58 \mathrm{dBi}$ at 38$\mathrm{GHz}$. It is important to mention that the single resonance at 36.5-GHz tuned from $L_{p}$ splits into dual-band by parametric analysis of $s$, which results in lowering the gain performance due to gain-bandwidth trade-off. The measured gain of $3 \times 3$ antenna array is above $7 \mathrm{dBi}$ in the bandwidth of each resonant band. The gain can be further improved with increase in number of the antenna elements. However, the proposed dimensions with $3 \times 3$ elements are compatible with integration in mobile handsets. Numerically computed results exhibit a reasonable efficiency of approx. $70 \%$ or above in all the MMW bands of the antenna.

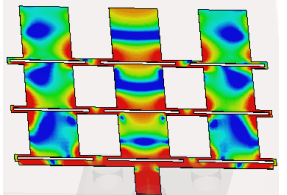

(a)

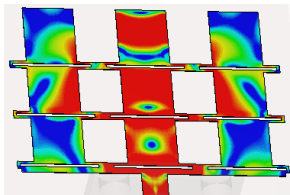

(b)

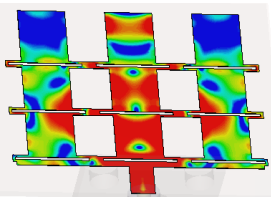

(c)
Fig. 6. Surface current distribution of the MMW 2D antenna array: (a) at 29$\mathrm{GHz}$; (b) at 33-GHz; (c) at 38-GHz.

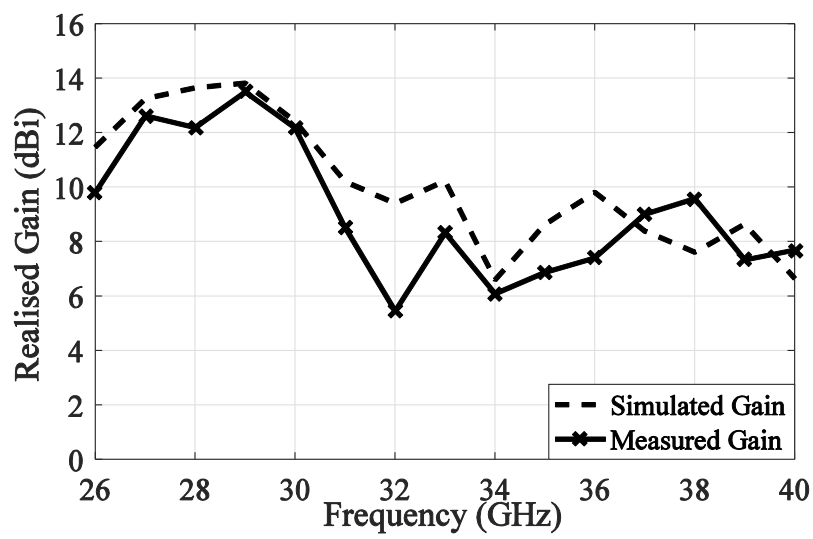

Fig. 7. Simulated and measured realized gain of the MMW 2D antenna array.

\section{CONCLUSION}

A novel and compact antenna array configuration has been presented and investigated aiming to operate at three significant MMW bands. The design principle of linear arrays has been diversified into a well-matched arrangement of two different radiating elements extended in a $2 \mathrm{D}$ plane. The standard theory of Franklin antenna suggests a high gain and directivity, but with the limitation of being narrowband single frequency operation. This work suggests a geometrical modification of design parameters to achieve multiband response with reasonable gain profile. Several parameters have been parametrically analyzed to achieve frequency tuning of the resonant bands. The performance analysis of the antenna has demonstrated that the objectives of achieving a triple-band response at $28-\mathrm{GHz}, 33-\mathrm{GHz}$, as well as $37-39$ $\mathrm{GHz}$ is accomplished with the peak gain of $13.5 \mathrm{~dB}$ at 29$\mathrm{GHz}$. The advantages of compactness, efficient design, and planar geometry have been successfully deployed, in order to attain high performance features which suggest the reported MMW 2D antenna array as a potential candidate for future 5G wireless communication systems and networks.

\section{REFERENCES}

[1] T. S. Rappaport, J. N. Murdock, and F. Gutierrez, "State of the art in 60 GHz integrated circuits \& systems for wireless communications," Proc. IEEE, vol. 99, no. 8, pp. 1390-1436, Aug. 2011.

[2] Z. Pi and F. Khan, "An introduction to millimeter-wave mobile broadband systems," IEEE Commun. Mag., vol. 49, no. 6, pp. 101-107, Jun. 2011.

[3] C. X. Wang et al., "Cellular architecture and key technologies for 5G wireless communication networks" IEEE Commun. Mag., vol. 52, no. 2, pp. 122-130, Feb. 2014.

[4] L. Wei, R. Q. Hu, Y. Qian, and G. Wu, "Key elements to enable millimeter wave communications for 5G wireless systems," IEEE Wireless Commun., vol. 21, no. 6, pp. 136-143, Dec. 2014.

[5] S. Rangan, T. S. Rappaport, and E. Erkip, "Millimeter-wave cellular wireless networks: potentials and challenges," Proc. IEEE, vol. 102, no. 3, pp. 366-385, Mar. 2014.

[6] Use of Spectrum Bands Above $24 \mathrm{GHz}$ for Mobile Radio Services, Federal Register, vol. 81, no. 219, Nov. 2016.

[7] M. M. M. Ali and A. R. Sebak, "Design of compact millimeter wave massive MIMO dual-band $(28 / 38 \mathrm{GHz})$ antenna array for future $5 \mathrm{G}$ communication systems," in Proc. 17 Int. Symp. Antenna Tech. Applied Electromagnetics (ANTEM), 2016, pp. 1-2.

[8] H. Chu and Y. X. Guo, "A filtering dual-polarized antenna subarray targeting for base stations in millimeter-wave $5 \mathrm{G}$ wireless communications," IEEE Trans. Components, Packaging Manufacturing Tech., 2017.

[9] S. X. Ta, H. Choo and I. Park, "Broadband printed-dipole antenna and its arrays for 5G applications," IEEE Antennas Wireless Propag. Lett., vol. 16, no. ,pp. 2183-2186, 2017.

[10] M. T. Passia, M. Nitas, and T. V. Yioultsis, "A fully planar antenna for millimeter-wave and 5G communications based on a new CSRRenhanced substrate-integrated waveguide," in Proc. Int. Workshop Antenna Tech.: Small Antennas, Innovative Structures, and Applications (iWAT), 2017, pp. 183-186.

[11] S. Ershadi et al., "Wideband subarray design for 5G an antenna arrays," in Proc. URSI Asia-Pacific Radio Science Conf. (URSI AP-RASC), 2016, pp. 185-187.

[12] A. Holub and M. Polivka, "Collinear microstrip patch antenna," in Passive Microwave Components and Antennas, InTech, Ch. 24, pp. 513-530, Apr. 2010.

[13] M. Polivka, A. Holub, and M. Mazanek, "Collinear microstrip patch antenna," Radioengineering, vol. 14, no. 4, pp. 40-42, 2005.

[14] M. Polivka and A. Holub, "Planar version of Collinear Microstrip Patch Antenna," International Conference on Microwaves, Radar \& Wireless Communications, 2006, pp. 959-962

[15] K. Solbach, "Microstrip-Franklin antenna," IEEE Trans. Antennas Propag., vol. 30, no. 4, pp.773-775, Jul. 1982.

[16] P. P. Wang, M. A. Antoniades, and G. V. Eleftheriades, "An investigation of printed Franklin antennas at X-band using artificial (metamaterial) phase-shifting lines," IEEE Trans. Antennas Propag., vol. 56, no. 10, pp. 3118-3128, Oct. 2008.

[17] S. H. Chang et al., "A Franklin array antenna for wireless charging applications," PIERS, vol. 6, no. 4, 2010.

[18] C. H. Kuo, C. C. Lin, J. S. Sun, "Modified microstrip Franklin array antenna for automotive short-range radar application in blind spot information system," IEEE Antennas Wireless Propag. Lett., 2017.

[19] Z. Peng et al., "An Effective Coverage Scheme With Passive-Reflectors for Urban Millimeter-Wave Communication," IEEE Antennas Wireless Propag. Lett., vol. 15, no. , pp. 398-401, 2016. 